BMJ Open

Diabetes

Research

\& Care

\title{
Pioglitazone and risk of mortality in patients with type 2 diabetes: results from a European multidatabase cohort study
}

\author{
Helen Strongman, ${ }^{1}$ Pasi Korhonen, ${ }^{2}$ Rachael Williams, ${ }^{1}$ Shahram Bahmanyar, ${ }^{3}$ \\ Fabian Hoti, ${ }^{2}$ Solomon Christopher, ${ }^{2}$ Maila Majak, ${ }^{2}$ Leanne Kool-Houweling, ${ }^{4}$ \\ Marie Linder, ${ }^{3}$ Paul Dolin, ${ }^{5}$ Edith M Heintjes ${ }^{4}$
}

To cite: Strongman $\mathrm{H}$, Korhonen P, Williams R, et al. Pioglitazone and risk of mortality in patients with type 2 diabetes: results from a European multidatabase cohort study. BMJ Open Diab Res Care 2017;5:e000364. doi:10.1136/ bmjdrc-2016-000364

Received 17 November 2016 Revised 27 March 2017 Accepted 3 April 2017

\section{CrossMark}

\footnotetext{
${ }^{1}$ Clinical Practice Research Datalink, London, UK ${ }^{2}$ EPID Research, Espoo, Finland

${ }^{3}$ Centre for

Pharmacoepidemiology, Karolinska Institute, Stockholm, Sweden ${ }^{4}$ PHARMO Institute, Utrecht The Netherlands ${ }^{5}$ Takeda Development Centre Europe, London, UK
}

Correspondence to Helen Strongman; helen.strongman@mhra. gov.uk

\section{ABSTRACT}

Objectives Estimate and compare the risk of mortality in patients whose antidiabetic therapy is modified to include pioglitazone compared with an alternative antidiabetic medication at the same stage of disease progression. Design Retrospective cohort study.

Setting Pooled analysis of clinical data collected from primary and/or secondary care settings in four European countries: Finland, The Netherlands, Sweden and the UK . Participants 56337 patients with type 2 diabetes mellitus first prescribed pioglitazone between 2000 and 2011, and 56337 patients never prescribed pioglitazone matched by treatment stage, history of diabetes, diabetes complications and cardiovascular disease, and year of cohort entry using exact and propensity score matching. Patients were followed-up for a mean of 2.90 (SD 2.21) and 2.83 (SD 2.37) years in the pioglitazone-exposed and non-pioglitazone-exposed groups, respectively.

Outcomes All-cause mortality ascertained from clinical or registry data. Mortality was a planned secondary outcome in a study primarily studying the association of pioglitazone use with bladder cancer risk.

Results The crude overall mortality rate per 10000 patient years was $206(95 \% \mathrm{Cl} 199$ to 213$)$ in the pioglitazone-exposed group and 448 (95\% $\mathrm{Cl} 438$ to 458$)$ in the non-pioglitazone-exposed group. The crude HR comparing pioglitazone to alternative antidiabetic exposure was $0.46(95 \% \mathrm{Cl} 0.45$ to 0.48$)$. This reduced in magnitude to $0.67(95 \% \mathrm{Cl} 0.64$ to 0.70 ) following further adjustment for matching variables, propensity scores, age, gender and time-dependent variables representing use of alternative antidiabetic drugs.

Conclusions In this large observational cohort study of patients with type 2 diabetes, pioglitazone exposure was associated with a statistically significant decrease in the risk of all-cause mortality across four European countries. Results should be interpreted with caution due to the potential for residual confounding.

Protocol registration European Network of Centres for Pharmacoepidemiology and Pharmacovigilance.

\section{INTRODUCTION}

Newly diagnosed adult patients with diabetes have 5-10 years shorter life expectancy than

\section{Significance of this study}

What is already known about this subject?

- Evidence from the large Prospective Pioglitazone Clinical Trial In Macrovascular Events (PROactive) and Insulin Resistance Intervention after Stroke (IRIS) trials indicates that pioglitazone did not increase or reduce the risk of death from any cause in patients with type 2 diabetes at high-risk of cardiovascular disease (PROactive) or prediabetic patients with a history of ischemic stroke or transient ischemic attack (IRIS).

- Observational studies and meta-analyses of smaller randomized trials studying the risk of cardiovascular events have demonstrated a statistically significant reduction in the risk of all-cause mortality with pioglitazone use.

What are the new findings?

- This pan-European, observational, retrospective large cohort study indicates that prescribing pioglitazone compared with an alternative treatment decision at the same stage of disease progression was associated with a statistically significant $33 \%$ reduction in all-cause mortality.

How might these results change the focus of research or clinical practice?

- This further supports the safe use of pioglitazone. Additional research is recommended to evaluate the association of pioglitazone use with all-cause mortality.

the general population ${ }^{12}$ with cardiovascular disease being the leading cause of death in this patient group. Randomized clinical trials (RCTs) demonstrate that antidiabetic medications including pioglitazone improve insulin sensitivity in insulin-resistant patients resulting in lower plasma glucose and insulin concentrations and lower HbAlc values. ${ }^{3-5}$ Use of pioglitazone in combination with sulfonylurea, metformin or insulin has an additive effect in patients with type 2 diabetes 
mellitus (T2DM) ${ }^{6-9}$ This reduction in HbA1c has been hypothesized to protect patients with T2DM from macrovascular complications and reduced life expectancy. ${ }^{10-13}$ Meta-analyses of randomized trials studying the risk of cardiovascular events have demonstrated a statistically significant reduction in the risk of all-cause mortality with pioglitazone use. ${ }^{14}{ }^{15}$ The more recent meta-analysis by Mannucci et al aimed to assess the association of pioglitazone treatment with risk of non-fatal coronary events, cardiovascular and all-cause mortality. ${ }^{15}$ The meta-analysis demonstrated a statistically significant association of pioglitazone with reduced all-cause mortality OR 0.30 (95\% CI 0.14 to 0.63 ) in trials restricted to relatively low-risk patients with T2DM. Comparators in these trials included placebo, sulphonylureas (SU), metformin, rosiglitazone and glitazars. The large PROspective pioglitAzone Clinical Trial In macroVascular Events (PROactive) trial, ${ }^{16}$ conducted in high-risk patients with pre-existing macrovascular disease and T2DM, found that pioglitazone directionally reduced the risk of the composite primary endpoint-death from any cause, non-fatal myocardial infarction (MI), stroke, acute coronary syndrome, leg amputation, coronary revascularization, or revascularization of the leg (HR $0.90,95 \%$ CI 0.80 to 1.02). However, pioglitazone use significantly reduced the main secondary endpoint of all-cause mortality, MI and stroke (HR $0.84,95 \%$ CI 0.72 to 0.98 ). Fewer deaths contributed to the primary and secondary endpoint in the pioglitazone arm than the placebo arm. An observational study using US claims data found a significantly lower risk of all-cause mortality in patients with T2DM prescribed pioglitazone compared with insulin (HR 0.33, 95\% CI 0.31 to 0.36$).{ }^{17}$ More recently, a cohort study using data from a UK primary care database showed a decreased risk of all-cause mortality (HR $0.82,95 \%$ CI 0.77 to 0.88 ), heart failure (HR $0.86,95 \%$ CI 0.78 to 0.95 ) and cardiovascular (CV) disease (HR $0.94,95 \%$ CI 0.88 to 1.00 ) with use of glitazones in T2DM compared with non-use of these agents. ${ }^{18}$ Pioglitazone was prescribed to $90 \%$ of glitazone users in the study as rosiglitazone was found to increase cardiovascular risk and withdrawn from the UK market in $2010 .^{19}$

The primary objective of this pan-European multidatabase cohort study was to investigate the risk of bladder cancer associated with pioglitazone use compared with other diabetic drugs at the same stage of treatment in patients with T2DM. Results were recently published. ${ }^{20}$ Here we present findings from the secondary outcomes: all-cause and bladder cancer-specific mortality.

\section{METHODS}

\section{Study design and setting}

This pan-European multidatabase retrospective cohort study used six non-overlapping datasets from four European countries: Finland (FIN), the Netherlands (NL), Sweden (SWE) and the United Kingdom (UK).
Data from the PHARMO Database Network and Clinical Practice Research Datalink (CPRD) were used in the NL and UK, respectively.

\section{Data sources}

Drug exposure data were based on prescription data in the NL general practitioner (GP), UK GP, and UK GP hospital (GP-HOSP) datasets, and on dispensing data in the FIN, SWE and NL HOSP pharmacy (PHARM) datasets. All-cause and bladder cancer-specific (International Statistical Classification of Diseases and Related Health Problems 10th Revision (ICD-10) code C67) mortality were measured in linked national death records in FIN, SWE, NL-PHARM-HOSP and UK GP-HOSP datasets. All-cause mortality was identified using GP medical records in the NL GP and UK GP datasets. Further data to identify the study cohort and adjust for covariates was obtained from cancer registries, hospital discharge records, migration records, and national diabetes registers where available. The study period start and end dates respectively ranged by dataset from 1 Jan 2000 to 1 July 2006 and from 31 December 2010 to 30 June 2011.

\section{Participants}

The study population included patients over the age of 40 with T2DM whose diabetic treatment regimen was altered to include pioglitazone or any other antidiabetic medication during the study period. Potential cohort entry dates (CEDs) were set as the date of the first-ever pioglitazone prescription for pioglitazone-exposed patients and the date of switching to or add-on (initiation) of any other antidiabetic medication for the non-pioglitazone-exposed group. CEDs were excluded if there were under 12 months' membership in the medication database, any indication of bladder cancer prior to the CED, or if patients were concurrently exposed to another thiazolidinedione.

\section{Matching and covariates}

Guidelines for prescription of antidiabetic drugs recommend a stepwise approach that takes into account the efficacy of each drug substance in controlling blood sugars and the different safety profiles. Pioglitazone is therefore 'channelled' to patients at a specific stage of diabetes and with other characteristics that affect their prognosis. The following approach was therefore taken in an attempt to eliminate channelling bias. ${ }^{21}$ Propensity scores (PSs) were estimated for each CED using a weighted logistic regression model with weights reciprocal to the number of CEDs per individual. The PS model included baseline binary and categorical variables that were likely to have a direct impact on treatment choice and could be measured in all datasets. These included: antidiabetic treatment immediately prior to CED, whether the treatment change at CED was an add-on or switch in therapy, use of thiazolidinediones prior to CED, duration of treated diabetes, number of different antidiabetic medications used prior to CED, history of MI, stroke, heart 
failure, and the following diabetic complications, retinopathy or maculopathy, severe lower limb complications, renal complications, ketoacidosis, and hyperosmolar or ketoacidotic coma-duration of prescription database membership and calendar year of CED.

Pioglitazone CEDs were 1:1 fixed ratio matched and 1:10 variable ratio matched to non-pioglitazone CEDs using the PS distance and the following exact matching variables: antidiabetic treatment immediately prior to CED, whether the treatment change at CED was an add-on or switch in therapy, and use of thiazolidinediones prior to CED. Non-pioglitazone exposed patients were removed from further matching when one of their CEDs was matched to a pioglitazone-exposed patient. The number of matched non-pioglitazone-exposed patients varied between patients and countries in the 1:10 matched cohort resulting in an imbalance in characteristics. The main analysis was therefore based on the 1:1 fixed ratio matched cohort. Each patient was followed-up from CED until date of death or censorship due to end of membership of the database, end of database coverage, start of other thiazolidinediones or 30 June 2011. Timevarying (TV) covariates were generated to improve the estimation of adjusted HR. New binary TV covariates for use of each alternative antidiabetic treatment class and comorbidity history were generated for inclusion or testing in the regression model. Use of antidiabetic treatment group other than pioglitazone and comorbidity history was categorized as ever versus never used and ever versus never occurred up to any given time period. Age categories were fixed at baseline.

\section{Statistical analysis}

A pooled analysis was performed using all six cohorts. Descriptive data and standardized differences comparing the pioglitazone-exposed and non-pioglitazone-exposed groups were presented in the primary manuscript. ${ }^{20}$ Crude all-cause and bladder cancer mortality rates were estimated with 95\% CIs using Poisson regression. HR were generated using a Cox proportional hazards $(\mathrm{PH})$ model including the ever versus never exposure variable and a categorical dataset variable. Adjusted models also included as covariates age, gender, antidiabetic medications, exact matching variables, PS quintiles, and all variables that were included in the PS. Further covariates were included as confounders if their inclusion resulted in a minimum of $10 \%$ change in the HR of primary exposure (ever vs never exposed to pioglitazone) when introduced into the crude model. The PH assumption was examined by inspection of the Kaplan-Meier curves. Adjusted HRs were stratified for potential effect modifiers including age, gender, duration of treated diabetes at CED, year of CED, use of thiazolidinediones (other than pioglitazone) prior to CED and history of renal complications and congestive heart failure (CHF) at CED. Primary analyses for each of the two mortality endpoints included all datasets in which the endpoint was recorded. A number of preplanned sensitivity analyses assessed the robustness of the results with respect to outcome, exposure and follow-up definitions and the inclusion of baseline smoking, baseline body mass index and TV HbA1c as covariates. These covariates were not available for the FIN and NL-HOSP populations and missing variables were treated as a separate category.

\section{Further information}

Further details about the study methodology are published in the $\mathrm{BMJ}^{20}$ and in the European Network of Centres for Pharmacoepidemiology and Pharmacovigilance (ENCePP) E-register of studies (study title 'Pan-European Multi-Database Bladder Cancer Risk Characterisation Study').

\section{RESULTS}

The complete 1:1 matched cohort included 56337 patients in both the pioglitazone-exposed and the non-exposed groups. Descriptive statistics reported previously for the primary bladder cancer analyses ${ }^{20}$ demonstrated good covariate balance within the variables used in the matching process. The standardized differences for the proportion of patients with CHF, early cohort entry (2000-2003), and higher duration of medication database membership before CED (5-6 and 7+ years) marginally exceeded 10 , the lower threshold that we set for good balance. Descriptive statistics for a selection of covariates are provided in table 1 . The number of antidiabetic treatments prescribed at CED was similar in both cohorts. The most commonly prescribed treatment regimens in the never exposed to pioglitazone group were insulin alone or in combination $(36.99 \%)$, metformin or SU in combination $(21.41 \%)$, or combinations of other antidiabetic drugs $(31.10 \%)$. Pioglitazone was prescribed in a variety of treatment regimens: most commonly in combination with metformin $(34.83 \%)$ or metformin and SU (24.25\%).

A total of 10513 deaths occurred during follow-up in the 1:1 matched cohort. There were 3370 deaths in the ever exposed to pioglitazone group during a mean follow-up time of 2.9 years, and 7143 deaths in the non-pioglitazone-exposed group during a mean follow-up time of 2.8 years. The crude all-cause mortality rate per 10000 person-years was 206 (95\% CI 199 to 213) for patients ever exposed to pioglitazone and 448 (95\% CI 438 to 458) for patients never exposed to pioglitazone. The Kaplan-Meier curve is consistent with the $\mathrm{PH}$ assumption (figure 1).

A statistically significant $33 \%$ reduction in all-cause mortality was observed for ever exposed to pioglitazone compared with never exposure to pioglitazone (adjusted HR $0.67,95 \%$ CI 0.64 to 0.70$)$. Observed reductions were statistically significant in five of the six datasets. Relative risk reduction ranged from $11 \%$ in the NL GP to $46 \%$ in Finland. In the 56337 patients in the pioglitazone cohort, 23123 and 6462 patients received pioglitazone for at least 18 and 48 months, respectively. Total cumulative 
Table 1 Distribution of a selection of matching and non-matching covariates at cohort entry

\begin{tabular}{|c|c|c|}
\hline & $\begin{array}{l}\text { PIO exposed } \\
n=56337 n(\%)\end{array}$ & $\begin{array}{l}\text { Non-PIO exposed } \\
n=56337 \mathrm{n}(\%)\end{array}$ \\
\hline \multicolumn{3}{|l|}{ Age at CED (years) } \\
\hline $40-49$ & $6728(11.94)$ & $5608(9.95)$ \\
\hline $50-59$ & $14881(26.41)$ & $12618(22.40)$ \\
\hline $60-69$ & $18366(32.60)$ & $17262(30.64)$ \\
\hline$\geq 70$ & $16362(29.04)$ & $20849(37.01)$ \\
\hline Range (min, max) & $(40,102)$ & $(40,106)$ \\
\hline Mean $( \pm S D)$ & $63.24(10.86)$ & $65.38(11.56)$ \\
\hline Median (Q1, Q3) & $63.00(55.03,71.00)$ & $65.00(57.00,74.00)$ \\
\hline \multicolumn{3}{|l|}{ Gender } \\
\hline Male & $31732(56.33)$ & $30561(54.25)$ \\
\hline Female & $24605(43.67)$ & $25776(45.75)$ \\
\hline \multicolumn{3}{|l|}{ Duration of treated diabetes at CED (years) } \\
\hline$<1$ & $7805(13.85)$ & $7883(13.99)$ \\
\hline $1-<2$ & $6943(12.32)$ & $6679(11.86)$ \\
\hline $2-<4$ & $11865(21.06)$ & $11578(20.55)$ \\
\hline $4-<6$ & $10936(19.41)$ & $11411(20.25)$ \\
\hline$\geq 6$ & $18788(33.35)$ & $18786(33.35)$ \\
\hline Range (min, max) & $(0.00,34.18)$ & $(0.00,24.79)$ \\
\hline Mean $( \pm S D)$ & $4.72(3.65)$ & $4.78(3.70)$ \\
\hline Median (Q1, Q3) & $4.12(1.76,6.97)$ & $4.19(1.82,6.97)$ \\
\hline \multicolumn{3}{|l|}{ Use of other TZD medications prior to CED } \\
\hline Never & $41847(74.28)$ & $41847(74.28)$ \\
\hline Ever & $14490(25.72)$ & $14490(25.72)$ \\
\hline \multicolumn{3}{|c|}{ Antidiabetic treatment immediately prior to CED* } \\
\hline Metformin only & $16526(29.33)$ & $16526(29.33)$ \\
\hline Sulphonylureas (SU) only & $6110(10.85)$ & $6110(10.85)$ \\
\hline Metformin and SU & $14277(25.34)$ & $14277(25.34)$ \\
\hline Insulin (only or in combination) & $2705(4.80)$ & $2705(4.80)$ \\
\hline Other drugs or combinations & $10062(17.86)$ & $10062(17.86)$ \\
\hline No treatment & $6657(11.82)$ & $6657(11.82)$ \\
\hline \multicolumn{3}{|c|}{ Number of different antidiabetic drug classes being used at CED $†$} \\
\hline 1 & $6905(12.26)$ & $9961(17.68)$ \\
\hline 2 & $29216(51.86)$ & $29767(52.84)$ \\
\hline 3 & $18446(32.74)$ & $15917(28.25)$ \\
\hline$>3$ & $1770(3.14)$ & $692(1.23)$ \\
\hline \multicolumn{3}{|l|}{ Antidiabetic treatment at CED $†$} \\
\hline Metformin only & $19622(34.83)$ & $3496(6.21)$ \\
\hline SU only & $7442(13.21)$ & $2418(4.29)$ \\
\hline Metformin and SU & $13659(24.25)$ & $12062(21.41)$ \\
\hline Insulin (only or in comb.) & $2595(4.61)$ & 20840 (36.99) \\
\hline Other drugs or comb. & $6114(10.85)$ & $17521(31.10)$ \\
\hline No treatment & $6905(12.26)$ & $0(0.00)$ \\
\hline \multicolumn{3}{|l|}{ Diabetic complications at CED } \\
\hline Diabetic retinopathy or maculopathy & $5747(10.20)$ & $5953(10.57)$ \\
\hline
\end{tabular}


Table 1 Continued

\begin{tabular}{lll}
\hline & $\begin{array}{l}\text { PIO exposed } \\
\mathbf{n = 5 6 3 3 7} \mathbf{n}(\%)\end{array}$ & $\begin{array}{l}\text { Non-PIO exposed } \\
\mathbf{n = 5 6 3 3 7} \mathbf{n}(\%)\end{array}$ \\
\hline Lower limb severe complications & $1308(2.32)$ & $1530(2.72)$ \\
\hline $\begin{array}{l}\text { Diabetic renal complications } \\
\text { Ketoacidosis }\end{array}$ & $4771(8.47)$ & $4870(8.64)$ \\
\hline $\begin{array}{l}\text { Hyperosmolar/ } \\
\text { ketoacidotic coma }\end{array}$ & $112(0.20)$ & $145(0.26)$ \\
\hline Other comorbidities at CED & $822(1.46)$ & $1280(2.27)$ \\
\hline $\begin{array}{l}\text { Myocardial infarction or stroke } \\
\text { Congestive heart failure }\end{array}$ & $6112(10.85)$ \\
Year at CED & $4676(8.30)$ & $3077(5.46)$ \\
\hline 2000-2003 & $1674(2.97)$ & $6485(11.51)$ \\
\hline 2004-2007 & & $20513(36.41)$ \\
$2008-2011$ & $3960(7.03)$ & $29339(52.08)$ \\
\hline
\end{tabular}

*Treatments initiated at CED are not included.

†Treatments initiated at CED are included. For the pioglitazone-exposed group, the listed treatments are in addition to pioglitazone.

CED, cohort entry date; PIO, pioglitazone; TZD, thiazolidinedione.

pioglitazone dose exceeded $14000 \mathrm{mg}$ for 22550 patients and $40000 \mathrm{mg}$ for 7226 patients. Observed reductions were statistically significant for all doses and durations of exposure. The HR estimates for all-cause mortality are presented in figures 2 and 3. No additional variables met the inclusion criteria for confounders. The adjusted model therefore included age, gender, antidiabetic medications, PS quintiles, and all variables that were included in the PS model. Full model results are presented in the supplementary appendix.

For patients with current pioglitazone exposure, there was a significant $60 \%$ reduction in all-cause mortality compared with never exposure (HR $0.40,95 \%$ CI 0.37 to 0.43 ). With regard to cumulative dose and duration of pioglitazone use, a similar significant reduction in mortality risk was observed for all exposure strata.

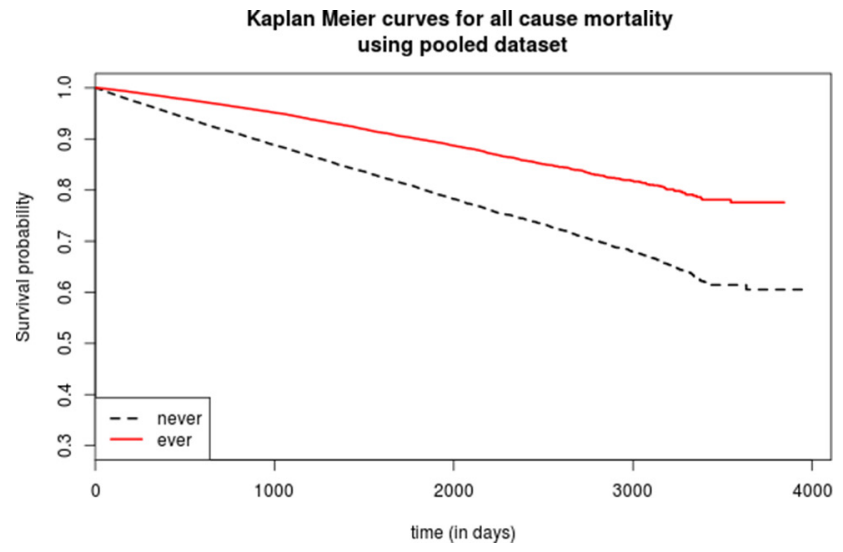

Figure 1 Kaplan-Meier plot of association of exposure to pioglitazone with all-cause mortality in patients with type 2 diabetes. Never: never exposed to pioglitazone. Ever: exposed to pioglitazone.
A statistically significant reduction in mortality risk was observed in all stratified and sensitivity analyses. The adjusted HR for patients prescribed and not prescribed thiazolidinedione prior to cohort entry was 0.85 (95\% CI 0.74 to 0.97 ) and 0.64 (95\% CI 0.61 to 0.68 ), respectively. This was the only stratified analysis in which the confidence intervals did not overlap (figure 4).

The pooled dataset for the bladder cancer mortality analysis included 34615 patients in the 1:1 matched pioglitazone and non-pioglitazone exposed groups. A total of 21 deaths due to bladder cancer occurred during follow-up including seven deaths in the pioglitazone-exposed group and 14 deaths in the non-pioglitazone-exposed group during a mean follow-up time of 2.7 years in both groups. The crude bladder cancer-specific mortality rate per 10000 person-years was 0.75 (95\% CI 0.36 to 1.57 ) for patients ever exposed to pioglitazone and 1.50 (95\% CI 0.89 to 2.54) for patients never exposed to pioglitazone. A statistically non-significant $39 \%$ reduction was observed in bladder cancer cause-specific mortality for ever exposed to pioglitazone compared with never exposed to pioglitazone (adjusted HR $0.61,95 \%$ CI 0.23 to 1.67 ). The adjusted model only included all the predefined covariates. Due to the small number of events, additional covariates or other exposure definitions were not considered.

\section{DISCUSSION}

Our analysis of this large pan-European retrospective cohort study found a statistically significant 33\% reduction in risk of all-cause mortality in patients with T2DM whose antidiabetic therapy was modified to include pioglitazone compared with an alternative antidiabetic medication at the same stage of disease progression. 
Category

\section{Pioglitazone exposure}

$\begin{array}{lr}\begin{array}{l}\text { never } \\ \text { ever }\end{array} & 7143(448.30) \\ \begin{array}{l}\text { Duration of } \\ \text { pioglitazone exposure } \\ \text { never }\end{array} & 3370(206.34) \\ <18 \text { months } & 7143(448.30) \\ 18-<48 \text { months } & 2171(212.08) \\ >48 \text { months } & 965(200.55) \\ \text { Cumulative } & 234(182.25) \\ \text { pioglitazone dose } & \\ \text { never } & \\ 1-14,000 \mathrm{mg} & 7143(448.30) \\ 14,001-40,000 \mathrm{mg} & 2187(215.64) \\ >40,000 \mathrm{mg} & 898(195.34) \\ & 285(178.87)\end{array}$

No. of events

(Incidence Rate)

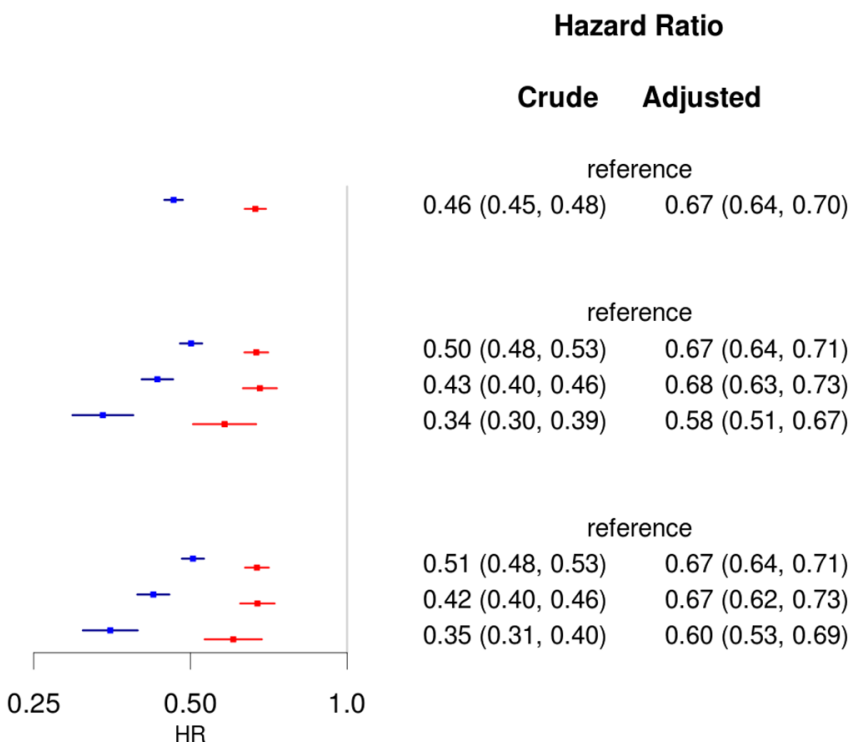

(1)

Figure 2 Number of events, the crude incidence rate, and the crude and adjusted HR with $95 \% \mathrm{Cl}$ for the association between pioglitazone use and all-cause mortality. Crude model: pioglitazone exposure variable and a dataset identifier. Adjusted model: Crude model plus, age, gender, use of metformin, use of sulphonylureas, use of insulin, and use of other antidiabetic drugs, all exact matching variables, propensity scores as quintiles, all propensity score variables evaluated at cohort entry date.

\section{All-cause mortality risk for ever vs. never exposed to Pioglitazone}

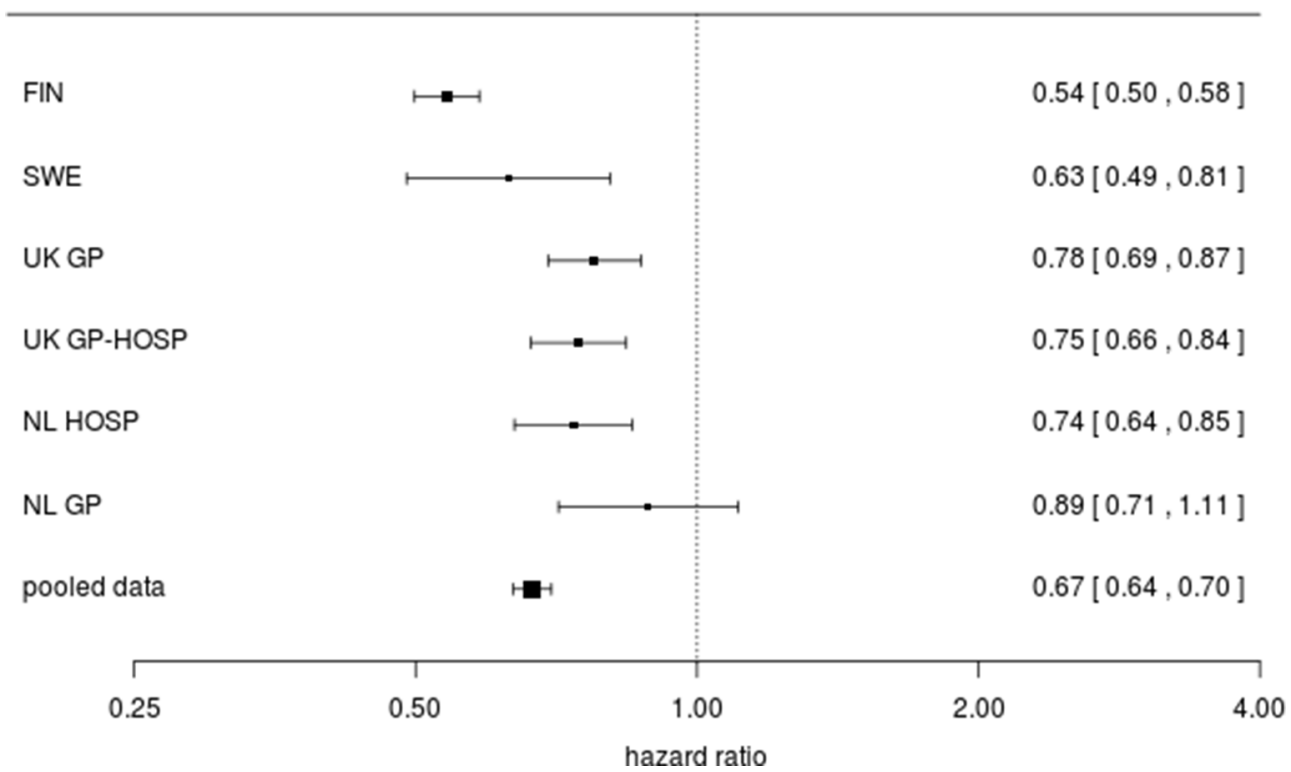

Figure 3 Adjusted HRs with $95 \% \mathrm{Cl}$ for the association between pioglitazone use and all-cause mortality for each dataset. The HRs and $95 \%$ Cls are presented in the original scale and the figure is plotted in the log scale. FIN, Finland; SWE, Sweden; United Kingdom GP, UK general practitioner; UK GP-HOSP, UK linked dataset; NL HOSP, Netherlands hospital data; NL GP, NL general practitioner. 


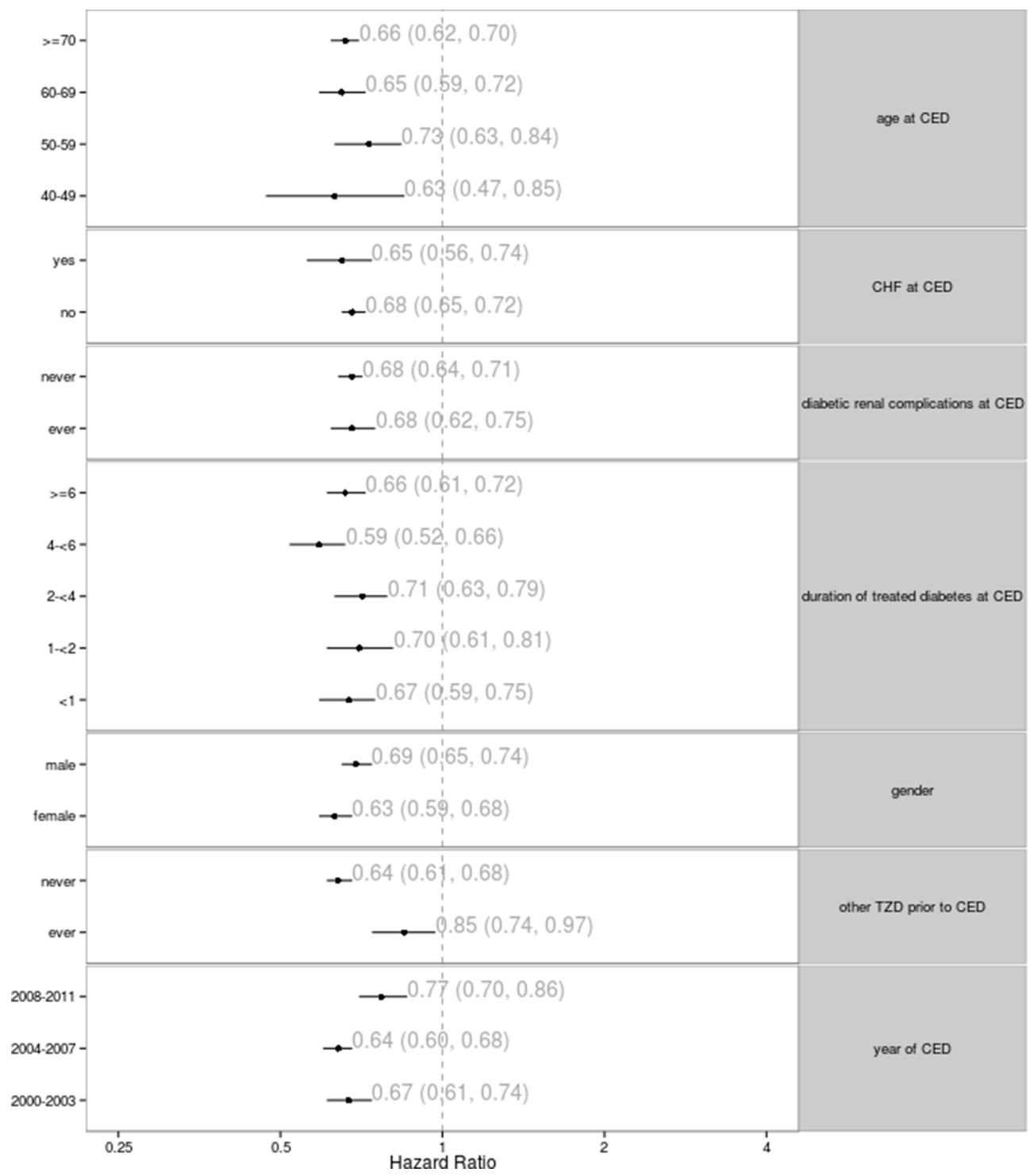

Figure 4 Adjusted $\mathrm{HRs}$ with $95 \% \mathrm{Cl}$ for the association between pioglitazone use and all-cause mortality stratified by key variables. CED, cohort entry date; CHF, congestive heart failure; TZD, thiazolidinedione.

Comparators included a full range of antidiabetic treatment regimens from metformin alone $(11 \%)$ to insulin used alone or in combination (36\%). Findings for bladder cancer-specific mortality estimated a similar $36 \%$ decrease with broad CIs consistent with a $77 \%$ decrease or a $67 \%$ increase, reflecting the small number of cases.

General strengths and weaknesses of the study have been reported previously. ${ }^{20}$ The cohort matching and regression modeling processes were designed for the primary outcome of incident bladder cancer. There is therefore a risk that important covariates influencing the association between pioglitazone exposure and all-cause mortality were not included. The most common cause of death in patients with T2DM is cardiovascular complications. ${ }^{22}$ Cardiovascular comorbidities were included in the PS, although good balance was not achieved for CHF, $2.97 \%$ and $5.46 \%$ in the pioglitazone exposed and unexposed cohorts, respectively. Stratifying the analysis by presence and absence of CHF at CED demonstrated similar reductions of all-cause mortality for the pioglitazone-exposed and non-pioglitazone-exposed groups. The imbalance is therefore unlikely to have biased the findings. Also, TV variables for cardiovascular comorbidities were included in the adjusted regression model. Variables such as socioeconomic status and general health may affect both choice of diabetic treatment and mortality risk. ${ }^{23}$ These were not included in the study protocol and would be difficult to measure consistently across datasets. They may therefore be residual confounders.

Observed risk reductions were statistically significant in five of the six datasets which supports the generalizability of our findings. The largest relative risk reduction was observed in Finland (HR 0.54, 95\% CI 0.50 to 0.58) and the smallest in the NL GP dataset (HR 0.89, 95\% CI 0.71 to 1.11 ). This may reflect differences in available data or in the care pathway for diabetes in these countries. All of the countries in this study have a publically funded national health service with limited co-pay for 
antidiabetic drugs during the study period. Nevertheless, the observed reduction in mortality risk may be due to inadequate or inappropriate prescribing in the comparator arm.

In agreement with our study, Mannucci's meta-analysis demonstrated a statistically significant association of pioglitazone use with reduced all-cause mortality (OR $0.30,95 \%$ CI 0.14 to 0.63 ) in patients at relatively low risk of cardiovascular events and death. ${ }^{15}$ The primary endpoint for the majority of RCTs was HbAlc. Patients included in the meta-analysis had a mean diabetes duration of 6.6 years and mean HbAlc of $8.1 \%$. This is similar to the mean 4.7 year duration of treated diabetes and mean HbAlc (where available) of $8.5 \%$ and $8.8 \%$ in the exposed and control arms, respectively, of our study. The major advantage of clinical trials compared with our study is that patients are randomized to treatment. This greatly reduces the risk of residual confounding compared with our observational study methodologies. However, trials included in the meta-analysis were generally of short duration (8-156 weeks) and counted zero or few mortality events (total 56). Our study had a mean follow-up of 2.9 years and counted a total of 10513 deaths. The results of our study are consistent with an observational cohort study that demonstrated a $23 \%$ reduction in all-cause mortality with use compared with non-use of glitazones (primarily pioglitazone) in the UK. ${ }^{18}$ There were 597 deaths in the study cohort over an average 4.5 years of follow-up time. Yang et $a l$ s observational study ${ }^{17}$ comparing first time users of insulin or pioglitazone in a US claims database also found a statistically significant decrease in risk of all-cause mortality in the pioglitazone cohort compared with the insulin cohort.

In contrast, the large PROactive trial found no statistically significant reduction in all-cause mortality with pioglitazone use compared with placebo in either the 3-year trial (HR $0.96,95 \% \mathrm{CI} 0.78$ to 1.18 ) or the 10 -year observational follow-up period (HR $0.93,95 \%$ CI 0.84 to 1.04$).{ }^{1624}$ This study was designed to study the effects of pioglitazone on cardiovascular disease in high-risk patients with pre-existing macrovascular disease and T2DM. Similar results were found in the Insulin Resistance Intervention after Stroke (IRIS) trial, which measured the effects of pioglitazone on cardiovascular disease and all-cause mortality in prediabetic patients with a recent history of ischemic stroke or transient ischemic attack. By 4.8 years, there was no statistically significant difference in all-cause mortality in the pioglitazone group compared with the placebo group (HR $0.93,95 \%$ CI 0.73 to 1.17 ).

Pioglitazone is an agonist of peroxisome proliferator-activated receptor $\gamma($ PPAR- $\gamma)$ indicated for treatment of T2DM. Data show that pioglitazone causes partial activation of PPAR- $\alpha$. These actions modulate the transcription of genes with favorable effects on fat distribution, lipid and protein metabolism, insulin sensitivity, plasma glucose, inflammation, and vascular endothelial function. ${ }^{25}$ There are several potential mechanisms that could underlie the association of pioglitazone and reduced mortality. The overall changes induced by pioglitazone suggest a general improvement in various risk factors that might reduce cardiovascular morbidity and mortality. ${ }^{16}$ Evidence also indicates that pioglitazone reduces the levels of various cardiovascular risk parameters/inflammatory markers, such as highly sensitive C-reactive protein and carotid intima-media thickness, independently of its effect on glycemic control. ${ }^{26}$ These mechanisms could be the link between treatment and reduced risk of macrovascular disease in patients with diabetes ${ }^{16}$ but more research is needed to confirm this view.

Evidence indicates that patients with T2DM have reduced life expectancy compared with the general population. ${ }^{1} 2$ RCTs provide limited and inconclusive evidence concerning the effect of pioglitazone on all-cause mortality.

\section{CONCLUSIONS}

This large, observational multidatabase European cohort study found that prescribing pioglitazone compared with an alternative treatment decision at the same stage of disease progression was associated with a reduction in all-cause mortality rate. Further observational and prospective studies that are specifically designed to test the association between pioglitazone use and all-cause mortality objective are required.

\section{Other information}

The European Medicines Agency (EMA) mandated the Marketing Authorization Holder of Actos (pioglitazone) to conduct this study. The study protocol was reviewed and approved by the EMA, and the study was granted the ENCePP Seal reflecting transparency and quality in research. The study was fully funded by Takeda Development Centre Europe Ltd.

Acknowledgments Dr Tim Williams (CPRD) and Susan Eaton (CPRD) were involved in the study design. Irene Bezemer (PHARMO Institute) contributed in analytical discussions and Eline Houben (PHARMO Institute) contributed to the construction of the analysis file. The Swedish National Diabetes Register provided detailed data for this study. We would like to thank all patients and participating staff who have contributed to the register.

Contributors All authors planned and designed the study. SC, MM, ML, EMH, LK$\mathrm{H}, \mathrm{RW}$ and $\mathrm{HS}$ performed the data management and data analysis, and all authors interpreted the data. All authors drafted the manuscript, revised the paper critically for important intellectual content and approved the final version of the manuscript. PK supervised the study and is the guarantor.

Competing interests All authors have completed the Unified CompetingInterest form at (available on request from the corresponding author) and declare that $\mathrm{PK}$, FH,SC, and MM are employed by EPID Research, EH is and LKH was employed by PHARMOInstitute, RW and HS are employed by CPRD, and ML and SB are employed byKarolinska Institute. EPID Research, PHARMO Institute, CPRD and Karolinskalnstitute perform commissioned pharmacoepidemiological studies and thus theiremployees have been and currently are working in collaboration with severalpharmaceutical companies (including Takeda). PD is employed by Takeda Patient consent This is a register-based study with anonymous data and no patient contact.

Provenance and peer review Not commissioned; externally peer reviewed. Data sharing statement Criteria and process for sharing the analytical countryspecific datasets and meta-analysis dataset for third parties is defined in the study protocol available at the ENCePP E-Register of Studies. 
Open Access This is an Open Access article distributed in accordance with the Creative Commons Attribution Non Commercial (CC BY-NC 4.0) license, which permits others to distribute, remix, adapt, build upon this work non-commercially, and license their derivative works on different terms, provided the original work is properly cited and the use is non-commercial. See: http://creativecommons.org/ licenses/by-nc/4.0/

(c) Article author(s) (or their employer(s) unless otherwise stated in the text of the article) 2017. All rights reserved. No commercial use is permitted unless otherwise expressly granted.

\section{REFERENCES}

1 Panzram G. Mortality and survival in type 2 (non-insulin-dependent) diabetes mellitus. Diabetologia 1987;30:123-31.

2 Roper NA, Bilous RW, Kelly WF, et al. Excess mortality in a population with diabetes and the impact of material deprivation: longitudinal, population based study. BMJ 2001;322:1389-93.

3 Rajagopalan S, Dutta P, Hota D, et al. Effect of low dose pioglitazone on glycemic control and insulin resistance in type 2 diabetes: a randomized, double blind, clinical trial. Diabetes Res Clin Pract 2015;109:e32-e35.

4 Miyazaki Y, Matsuda M, DeFronzo RA. Dose-response effect of pioglitazone on insulin sensitivity and insulin secretion in type 2 diabetes. Diabetes Care 2002;25:517-23.

5 Abdul-Ghani MA, Puckett C, Triplitt C, et al. Initial combination therapy with metformin, pioglitazone and exenatide is more effective than sequential add-on therapy in subjects with new-onset diabetes. results from the Efficacy and durability of initial combination therapy for type 2 Diabetes (EDICT): a randomized trial. Diabetes Obes Metab 2015;17:268-75.

6 Einhorn D, Rendell M, Rosenzweig J, et al. Pioglitazone hydrochloride in combination with metformin in the treatment of type 2 diabetes mellitus: a randomized, placebo-controlled study. the pioglitazone 027 Study Group. Clin Ther 2000;22:1395-409.

7 Matthews DR, Charbonnel BH, Hanefeld M, et al. Long-term therapy with addition of pioglitazone to metformin compared with the addition of gliclazide to metformin in patients with type 2 diabetes: a randomized, comparative study. Diabetes Metab Res Rev $2005 ; 21: 167-74$

8 Charbonnel B, Schernthaner G, Brunetti P, et al. Long-term efficacy and tolerability of add-on pioglitazone therapy to failing monotherapy compared with addition of gliclazide or metformin in patients with type 2 diabetes. Diabetologia 2005;48:1093-104.

9 Ceriello A, Johns D, Widel M, et al. Comparison of effect of pioglitazone with metformin or sulfonylurea (monotherapy and combination therapy) on postload glycemia and composite insulin sensitivity index during an oral glucose tolerance test in patients with type 2 diabetes. Diabetes Care 2005;28:266-72.

10 Mannucci E, Dicembrini I, Lauria A, et al. Is glucose contro important for prevention of cardiovascular disease in diabetes? Diabetes Care 2013;36(Suppl 2):S259-S263.
11 Li W, Katzmarzyk PT, Horswell R, et al. HbA1c and all-cause mortality risk among patients with type 2 diabetes. Int $\mathrm{J}$ Cardiol 2016;202:490-6.

12 Arnold LW, Wang Z. The HbA1c and all-cause mortality relationship in patients with type 2 diabetes is $\mathrm{J}$-shaped: a meta-analysis of observational studies. Rev Diabet Stud 2014;11:138-52.

13 Ekström N, Svensson AM, Miftaraj M, et al. Cardiovascular safety of glucose-lowering agents as add-on medication to metformin treatment in type 2 diabetes: report from the Swedish National Diabetes Register. Diabetes Obes Metab 2016;18:990-8.

14 Lincoff AM, Wolski K, Nicholls SJ, et al. Pioglitazone and risk of cardiovascular events in patients with type 2 diabetes mellitus: a meta-analysis of randomized trials. JAMA 2007;298:1180-8.

15 Mannucci E, Monami M, Lamanna C, et al. Pioglitazone and cardiovascular risk. A comprehensive meta-analysis of randomized clinical trials. Diabetes Obes Metab 2008;10:1221-38.

16 Dormandy JA, Charbonnel B, Eckland DJ, et al. Secondary prevention of macrovascular events in patients with type 2 diabetes in the PROactive Study (PROspective pioglitAzone clinical trial in macroVascular events): a randomised controlled trial. Lancet 2005;366:1279-89.

17 Yang J, Vallarino C, Bron M, et al. A comparison of all-cause mortality with pioglitazone and insulin in type 2 diabetes: an expanded analysis from a retrospective cohort study. Curr Med Res Opin 2014;30:2223-31.

18 Hippisley-Cox J, Coupland C. Diabetes treatments and risk of heart failure, cardiovascular disease, and all cause mortality: cohort study in primary care. BMJ 2016;354:i3477.

19 Medicines and Healthcare products Regulatory Agency. Rosiglitazone: recommended withdrawal from clinical use. 2010 https://www.gov.uk/drug-safety-update/rosiglitazonerecommended-withdrawal-from-clinical-use.

20 Korhonen P, Heintjes EM, Williams R, et al. Pioglitazone use and risk of bladder cancer in patients with type 2 diabetes: retrospective cohort study using datasets from four European countries. BMJ 2016;354:i3903.

21 Petri $\mathrm{H}$, Urquhart J. Channeling Bias in the interpretation of drug effects. Stat Med 1991;10:577-81.

22 Morrish NJ, Wang SL, Stevens LK, et al. Mortality and causes of death in the WHO multinational study of vascular disease in Diabetes. Diabetologia 2001;44(Suppl 2):S14-S21.

23 Brown AF, Ettner SL, Piette J, et al. Socioeconomic position and health among persons with diabetes mellitus: a conceptual framework and review of the literature. Epidemiol Rev 2004;26:63-77.

24 Erdmann E, Harding S, Lam H, et al. Ten-year observational followup of PROactive: a randomized cardiovascular outcomes trial evaluating pioglitazone in type 2 diabetes. Diabetes Obes Metab 2016;18:266-73.

25 Kernan WN, Viscoli CM, Furie KL, et al. Pioglitazone after ischemic stroke or transient ischemic attack. $N$ Engl $\mathrm{J} \mathrm{Med}$ 2016;374:1321-31.

26 Pfützner A, Marx N, Lübben G, et al. Improvement of cardiovascular risk markers by pioglitazone is independent from glycemic control: results from the pioneer study. J Am Coll Cardiol 2005;45:1925-31. 